Mesurer le développement de la mémoire de travail : Exemple d'une tâche d'empan complexe adaptative

\title{
Measuring the development of working memory capacity: Example of an adaptive complex span task
}

Warning : The manuscript has not been peer-reviewed.

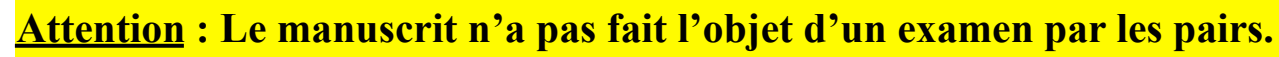

\author{
Alexandre Aubry 1 \\ Université de Picardie Jules Verne \\ Corentin Gonthier ${ }^{2}$ \\ Université Rennes 2 \\ Mathieu Hainselin ${ }^{1}$ \\ Université de Picardie Jules Verne \\ Béatrice Bourdin ${ }^{1}$ \\ Université de Picardie Jules Verne
}

${ }^{1}$ CRP-CPO EA 7273, Université de Picardie Jules Verne, Amiens, France

2 LP3C EA 1285, Université Rennes 2, Rennes, France

La correspondance concernant cet article devra être adressée à Béatrice Bourdin, CRPCPO EA7273, Chemin du Thil, 80025 Amiens, France

E-mail: beatrice.bourdin@u-picardie.fr 


\begin{abstract}
The aim of this current article is to provide a reflection on how to estimate working memory capacity (WMC), and to investigate its developmental trajectory from eight to twenty-three years-old with a single adaptive and multimodal task. WMC is defined as both storage and manipulation of the information during a cognitive activity. This cognitive aptitude is crucial for the learning and play an important role in the intellectual functioning. WMC is strongly related to some cognitives activities such as the language comprehension, the problems solving or the mathematics. The assessment of WMC therefore seems to be essential throughout lifespan. Although WMC is domain-general, most assessments WMC are based on verbal tasks only. Furthermore, WMC measurement in the developmental studies is limited by the need to preserve discriminating power at all possible ages. The use of a short and composite complex span task adapting itself to the individual's performance seems meet both requirements. Thus, we created a computerized task that is both multimodal (with verbal and nonverbal subtests) and adaptive to the individual's abilities: the Adaptive Composite Complex Span (ACCES). With a sample of 724 participants aged between 8 and 23 years-old, our findings show ACCES retains high discriminating power at all chronological ages with no ceiling effect or floor effect. Theoretical and clinical implication are discussed.
\end{abstract}

\title{
Keywords
}

Working Memory ; Adaptive testing ; Complex Span ; Developmental trajectory 


\section{Résumé}

Les objectifs de cet article sont d'apporter une réflexion sur la manière d'estimer la capacité de la mémoire de travail (MDT), et d'étudier son développement de 8 à 23 ans à partir d'une unique épreuve adaptative et multimodale. La MDT, définie comme le stockage et la manipulation simultanés de l'information, est essentielle pour les apprentissages et joue un rôle majeur dans le fonctionnement intellectuel. Elle est également importante dans de nombreuses activités cognitives, comme la compréhension, la résolution de problèmes ou encore les mathématiques. Il est donc essentiel d'évaluer la capacité de la MDT au travers du développement. Alors que la capacité en MDT est indépendante du mode de présentation, la plupart des épreuves évaluant la capacité de la MDT concernent uniquement la modalité verbale, induisant un biais dans son estimation. Par ailleurs, dans les études développementales, l'évaluation de la capacité de la MDT est fortement limitée par les contraintes liées à la sensibilité des épreuves aux différents âges des participants. L'utilisation d'une épreuve brève d'empan complexe unique s'adaptant aux performances de l'individu semble répondre à ces deux exigences. Ainsi, nous avons créé une épreuve informatisée, multimodale (verbale et non verbale), s'adaptant aux capacités de l'individu : l'Adaptive Composite Complex Span (ACCES). À partir d'un échantillon de 724 participants âgés de 8 ans à 23 ans, nos résultats montrent qu'ACCES est sensible à l'âge sans présenter d'effets plancher ni plafond. Les implications sont discutées en fonction des conceptions contemporaines de la MDT.

\section{Mots-Clés}

Mémoire de travail ; Test adaptatif ; Empan Complexe, Trajectoire développementale 
La mémoire de travail (MDT) peut être définie comme la capacité à stocker, manipuler et traiter des informations au cours d'activités cognitives. Le nombre d'informations pouvant être maintenues en MDT au cours d'une activité cognitive est limité (Wilhelm, Oberauer, Hildebrandt, \& Conway, 2013). Cette capacité joue un rôle essentiel dans le fonctionnement intellectuel (Ackerman, Beier, \& Boyle, 2005 ; Chuderski, 2013 ; Cornoldi \& Giofrè, 2014) et dans la réussite scolaire et les capacités d'apprentissage (e.g. Alloway \& Alloway, 2010 ; Gathercole, Brown, \& Pickering, 2003). La capacité en MDT contribue également au développement de nombreux processus cognitifs comme le langage (Linck, Osthus, Koeth, \& Bunting, 2013), ou la résolution de problèmes mathématiques (Swanson, 2011).

La plupart des chercheurs s'accordent pour reconnaître que la MDT naît de l'interaction de plusieurs processus cognitifs. La manière dont est décrit et théorisé le concept de MDT varie en fonction des modèles (Baddeley, 2012 ; Barrouillet, Bernardin, \& Camos, 2004 ; Cowan, 2001 ; Engle et al., 1999 ; Logie, 2011). Cependant, quelle que soit la manière dont nous conceptualisons la MDT, il y a un large consensus relatif aux processus cognitifs impliqués dans la capacité de la MDT. Plus précisément, la MDT comprend deux processus cognitifs principaux : un processus mnésique, correspondant au rappel de l'information brièvement stockée, et un processus cognitif lié au traitement, à la manipulation et l'intégration de l'information. La combinaison de ces deux processus cognitifs permet ainsi de distinguer le concept de MDT de celui de mémoire à court terme (MCT), envisagé comme un simple système de stockage.

Globalement, la capacité de la MDT augmente de l'enfance à l'adolescence (Gathercole, 1999 ; Siegel \& Ryan, 1989) jusqu'à l'âge adulte et décline au cours du vieillissement (Alloway \& Alloway, 2013 ; Swanson, 1999). Cependant, l'augmentation linéaire des performances avec l'âge est sujet à discussion (Roberts, Strait, \& Decker, 2018). Certaines recherches ont observé un palier se situant au début de l'adolescence (Gathercole, 1999 ; Thaler et al., 2015), tandis que d'autres ne mettent en évidence aucun plateau, mais une augmentation continue de la capacité de la MDT (Goldstein et al., 2014). Ces paliers de progression peuvent cependant constituer un artefact lié au type de tâche ou à la méthode de cotation utilisés pour l'évaluation de la capacité de la MDT. En effet, le choix des épreuves d'évaluation de la MDT s'avère être un réel défi dans les études développementales. Des contraintes liées à la sensibilité des épreuves à tous les âges peuvent poser de nombreux 
problèmes impliquant la sous-estimation de la capacité de la MDT. En effet, une épreuve qui présente une sensibilité adéquate pour de jeune enfants peut être trop simple pour des enfants plus âgés, ce qui rend difficile l'utilisation de la même épreuve à des âges différents. De plus, la majorité des recherches développementales utilise uniquement des tests de MDT dans la modalité verbale (Alloway \& Alloway, 2013). Par conséquent, la capacité de la MDT est fortement affectée par des processus spécifiques liés à la tâche, comme les capacités linguistiques. La MDT peut être vue comme une aptitude cognitive générale issue de la corrélation forte entre les différentes tâches de MDT verbales et non verbales (Oswald, McAbee, Redick, \& Hambrick, 2014). Cette aptitude générale de MDT correspond à la capacité de traiter, de stocker et de rappeler des informations en cours, quelle que soit la modalité de cette information. Enfin, le type de cotation des épreuves de MDT varie selon les études et a un impact sur la fidélité et la validité de l'estimation de la capacité de la MDT (Conway et al., 2005 ; St Clair-Thompson \& Sykes, 2010). La question de la meilleure manière d'évaluer de la capacité de la MDT semble donc essentielle afin d'étudier son développement.

\section{Évaluation de la capacité de la mémoire de travail}

En général, la capacité de la MDT est évaluée à travers des tâches d'empans simples, comme l'empan de chiffres endroit/envers ; c'est notamment le cas dans les batteries d'évaluation des aptitudes cognitives comme le Wechsler Intelligence Scale for Children 5e Édition (WISC-V ; Wechsler, 2016), le Kaufman Assessment Battery for Children — 2de Édition (KABC-II ; Kaufman \& Kaufman, 2008) ou encore la NEPSY-2 (Korkman, Kirk, \& Kemp, 2012). La littérature s'accorde pourtant à dire que ces épreuves ne sont pas adaptées et ne mesurent pas réellement la capacité de la MDT (Conway et al., 2005; Delaloye, Ludwig, Borella, Chicherio, \& de Ribaupierre, 2008). En effet, lorsqu'il s'agit de rappeler une liste de chiffres à l'envers, la manipulation mentale demandée ne constitue pas un traitement cognitif suffisamment coûteux pour faire intervenir la MDT (Hutton \& Towse, 2001 ; St ClairThompson \& Allen, 2013 ; St Clair-Thompson \& Sykes, 2010). De plus, certaines de ces batteries associent, dans un seul indice composite, des épreuves évaluant la MCT et la MDT. Il y a donc une confusion entre ces deux types de mémoire. Par exemple, le score standard de l'épreuve Mémoire des Chiffres (WISC-V ; Wechsler, 2016) est constitué à partir des 
performances de l'individu à trois épreuves différentes de mémorisation de chiffres : la mémorisation de chiffres en ordre direct (évaluant la MCT), la mémorisation de chiffres en ordre indirect (supposée évaluer la MDT, mais constituant plutôt une mesure de MCT), et l'épreuve de séquence lettres-chiffres (supposée mesurer la MDT).

Dans le domaine de la recherche en psychologie cognitive, les performances en MDT sont généralement évaluées par des épreuves d'empan complexe (Conway et al., 2005). Ce type d'épreuve est constitué d'une alternance entre des phases de traitement, comme le jugement de la véracité d'une phrase, et des phases de mémorisation d'un stimulus, tel qu'un chiffre. À la fin de chaque série, l'individu doit rappeler les différents stimuli mémorisés dans l'ordre de présentation. Ce type de tâche impose un traitement cognitif suffisamment coûteux pour que les deux fonctions principales (stockage et traitement) de la MDT soient mobilisées. La structure d'une tâche d'empan complexe répond donc parfaitement à la définition classique de la MDT.

Par ailleurs, le temps de traitement de la phase interférente semble jouer un rôle important sur la capacité de rappel durant une tâche de MDT (Barrouillet \& Camos, 2012 ; Bayliss, Jarrold, Baddeley, Gunn, \& Leigh, 2005 ; Towse \& Hitch, 1995 ; Saito \& Miyake, 2004). En effet, chaque item mémorisé est susceptible d'être oublié à cause du temps passé sur la tâche interférente (Barrouillet, Bernadin, \& Camos, 2004 ; Camos \& Barrouillet, 2014 ; Cowan, 1999 ; Towse \& Hitch, 1995). Le temps de traitement de la tâche interférente est tributaire du coût du traitement de cette tâche (Barrouillet et al., 2004). Autrement dit, plus le coût de traitement de la phase interférente est faible, plus elle sera traité rapidement par l'individu facilitant ainsi la mémorisation des items. Par conséquent, l'estimation de la capacité de la MDT est influencée par le temps entre les items à mémoriser. Il est donc important de contrôler le temps entre les phases de mémorisation et de s'assurer de la réalisation de cette tâche concourante. Le contrôle de cette phase interférente a également une influence forte sur le lien entre la capacité de la MDT et les aptitudes cognitives comme le raisonnement fluide (Friedman \& Myiake, 2004 ; Lépine, Barrouillet, \& Camos, 2005 ; St Clair-Thompson, 2007). Ainsi, les tâches d'empan complexe fonctionnent mieux lorsque le temps des phases interférentes est contraint (Engle, Cantor, \& Carullo, 1992 ; McCabe, 2010). Les tâches informatisées permettent d'automatiser facilement l'alternance entre la phase interférente et la phase de mémorisation et de contrôler facilement le temps et la précision du traitement de 
la phase interférente, ce qui en fait de meilleures mesures de la capacité de la MDT (Conway et al., 2005 ; Gonthier, Thomasin, \& Roulin, 2015 ; Unsworth, Heitz, Schrock, \& Engle, 2005).

Il existe peu d'épreuves d'empan complexe disponibles en langue française. Des épreuves d'empan de lecture ont été développées en français (par ex., Delaloye et al., 2008 ; Desmette, Hupet, Schelstraete, \& van der Linden, 1995 ; Fournet et al., 2012 ; Seigneuric, De Guibert, Megherbi, Potier, \& Picard, 2009), mais elles permettent une évaluation de la capacité de la MDT uniquement dans la modalité verbale. L'utilisation d'une épreuve avec une seule modalité a pour conséquence d'influencer l'estimation de la capacité de la MDT par la variance spécifique de la tâche. Afin d'estimer le domaine général de la MDT, il est donc plus approprié d'utiliser des épreuves d'empan complexe composites, c'est-à-dire associant plusieurs modalités (par ex. la mémorisation de matériel verbal et spatial ; Foster et al., 2014). La première épreuve composite d'empans complexes à avoir été développée en langue française, intitulée Empan Complexe Composite (CCS ; Gonthier et al., 2015), était toutefois destinée à l'évaluation chez l'adulte. L'objectif du présent article est de présenter une adaptation de cette épreuve de mémoire de travail composite pour les enfants et adolescents intitulés Adaptive Composite Complex Span (ACCES, Gonthier, Aubry, \& Bourdin, 2017).

L'ACCES présente un avantage pour les études développementales grâce à sa procédure adaptative de la difficulté des essais, c'est-à-dire que le nombre d'items à retenir à l'essai suivant est déterminé par le nombre d'items correctement rappelés à l'essai précédent. Contrairement à une procédure qui présente les items dans l'ordre de difficulté ascendante, une procédure adaptative permet d'éviter qu'une même épreuve utilisée pour des enfants de 8 ans soit trop facile pour des adolescents de 16 ans et entraîne un effet plafond, ou à l'inverse, qu'une épreuve conçue pour des adolescents entraîne un effet plancher chez des enfants plus jeunes. Par ailleurs, les épreuves non-adaptatives ne permettent pas toujours d'évaluer correctement tous les individus, même au sein d'un même groupe d'âge (Vock \& Holling, 2008). À titre d'exemple, les enfants à haut potentiel intellectuel présentent généralement des performances en MDT plus élevées que celles des enfants typiques de même âge chronologique (Calero, García-Martín, Jiménez, Kazén, \& Araque, 2007). Ainsi, l'utilisation d'une procédure adaptative, intéressante pour l'ensemble de la population, quel que soit l'âge, semble particulièrement adaptée dans les études développementales, y compris 
avec des groupes d'individus ayant des capacités cognitives extrêmes (McCoach, Rambo, \& Welsh, 2012).

Par contraste, la plupart des épreuves de MDT utilisent une procédure ascendante pour la difficulté des essais, combinée à un critère d'arrêt. Autrement dit, l'individu devra réaliser des essais faciles, puis de plus en plus difficiles avant de parvenir aux essais plus adaptés à ses réelles performances. L'arrêt de l'épreuve est programmé après un certain nombre d'échecs consécutifs fixé à l'avance (e.g. Barrouillet, Gavens, Vergauwe, Gaillard, \& Camos, 2009). Ce type de procédure pose toutefois plusieurs problèmes, notamment en ce qui concerne la durée de passation pour les enfants plus âgés ou ayant une grande capacité de MDT, comme les enfants à haut potentiel intellectuel (Aubry \& Bourdin, 2017). Cela soulève notamment la question de la fatigue et de l'accumulation de l'interférence proactive en cours de tâche (Kane \& Engle, 2000). La présence d'une interférence proactive peut diminuer la puissance prédictive de la tâche de MDT (Lustig, May, \& Hasher, 2001), et la résistance à cette interférence proactive est dépendante de l'âge du participant (Loosli, Rahm, Unterrainer, Weiller, \& Kaller, 2014). Avec une procédure ascendante de la difficulté, la performance en MDT n'est pas estimée à partir du même nombre d'essais selon le niveau d'aptitude en MDT des participants. En effet, un participant ayant une capacité de MDT élevée peut échouer à un essai facile par inattention ou par ennui. Il peut donc avoir une sous-estimation critique de sa performance en MDT. L'utilisation d'une procédure adaptative permet de résoudre ces problèmes, dans la mesure où chaque individu réalise le même nombre d'essais et où la tâche n'est jamais trop éloignée de son niveau d'aptitude réel.

La méthode de cotation a également une importance cruciale dans l'appréhension de la variabilité interindividuelle des performances aux épreuves de MDT (Conway et al., 2005 ; St Clair-Thompson \& Sykes, 2010), et influence les caractéristiques psychométriques et prédictives des épreuves de MDT. Dans la littérature, nous retrouvons principalement deux types de cotation :

1) La cotation absolue correspond à la somme de tous les essais où tous les items ont été entièrement rappelés dans l'ordre d'apparition. Par exemple, l'expérimentateur présente une série de chiffres telle que 6-4-3-9. Dans ce type de cotation, l'enfant obtiendra soit 0 point si la réponse donnée comprend une ou plusieurs erreurs (par ex. : 6-3-4-9) soit 1 point si la réponse donnée est totalement correcte (par ex. : 6-4-3-9). La cotation absolue 
est souvent imposée par les batteries d'évaluation classiquement utilisées par les psychologues cliniciens, mais elle génère une perte d'informations importante. Par exemple, un enfant rappelant la séquence 6-4-3 a le même score qu'un enfant qui ne rappelle aucun chiffre, mais ils n'ont pas une performance équivalente en MDT. De ce fait, cette méthode a tendance à diminuer la fiabilité de la mesure de la capacité de la MDT (Conway et al., 2005 ; Redick et al., 2012) ainsi que l'utilité de cette mesure pour prédire les performances scolaires et les aptitudes cognitives telles que le raisonnement fluide (St Clair-Thompson, \& Sykes, 2010).

2) La cotation partielle correspond à la somme des items correctement rappelés. Par exemple, pour la série 6-4-3-9, le participant obtiendra 2 points s'il répond 6-3-4-9 et 4 points s'il restitue la série complète (par ex. : 6-4-3-9). Ce type de cotation permet de mieux prendre en compte la variabilité interindividuelle que la cotation absolue. Elle permet également d'améliorer la fidélité de la mesure ainsi que la prédiction des performances scolaires et des aptitudes cognitives de haut niveau (Conway et al., 2005 ; Redick et al., 2012 ; St Clair-Thompson \& Sykes, 2010). Ce mode de cotation a donc été retenu pour l'ACCES.

En synthèse, l'ACCES (Gonthier et al., 2017) a été développé de façon à tenir compte de plusieurs contraintes pour optimiser l'estimation de la capacité de la MDT dans une perspective développementale : une tâche informatisée, une évaluation à l'aide d'empans complexes, une combinaison de différentes tâches évaluant plusieurs modalités, une procédure adaptative et une cotation partielle des performances en rappel. L'un des intérêts de ce format est de pouvoir utiliser une même épreuve d'empans complexes abrégés au travers des différents groupes d'âge. La section suivante présente le détail de la composition de l'ACCES et un exemple, afin d'illustrer la trajectoire développementale de la capacité générale de la MDT. Nous nous intéresserons également au rôle de l'accroissement du temps de traitement des phases interférentes avec l'âge sur la capacité de la MDT. Nous discuterons ensuite de l'intérêt d'adapter une telle tâche informatisée pour les psychologues cliniciens en général et pour les psychologues scolaires en particulier. 


\section{MÉTHODE}

\section{Participants}

753 participants âgés de 7 ans et 9 mois à 23 ans et 10 mois ont participé à l'étude. Tous les enfants et adolescents étaient scolarisés en milieu ordinaire, du CE2 à la seconde. Ils ont été recrutés dans 12 établissements scolaires publics et privés de l'Académie d'Amiens avec une démographie socio-économique variée. Les participants n'avaient aucun trouble neurologique ou spécifique des apprentissages avéré. Une partie de l'échantillon a été utilisée pour l'étude de la validation de la tâche ACCES (Gonthier et al., 2017). Parmi notre échantillon, 177 étudiants de Licence ont été recrutés dans un département de Psychologie d'une université française.

\section{Matériel}

Mémoire de travail : Une épreuve de trois tâches d'empan complexe courtes et informatisées intitulée Adaptative Composite Complex Span (ACCES ; Gonthier et al., 2017) a été utilisée afin de mesurer le domaine général de la capacité de la MDT. L'épreuve a été programmée sous PsychoPy (Peirce, 2007). Elle présente une fiabilité test-retest acceptable $(r=0,70)$, et une corrélation avec les Matrices Progressives de Raven $(r=0,34)$ indiquant une validité concourante satisfaisante (Gonthier et al., 2017). L'épreuve est composée de 3 tâches classiques d'empan complexe abrégées : l'empan de lecture (Reading span), l'empan de symétrie (Symmetry span) et l'empan d'opération (Operation span). Toutes ces tâches ont la même structure (voir Figure 1) : les participants doivent résoudre des problèmes simples et mémoriser des stimuli présentés après chaque problème. À la fin de chaque essai, les participants sont invités à rappeler tous les stimuli mémorisés dans l'ordre d'apparition. L'ACCES utilise une procédure adaptative de la difficulté des essais selon les performances du participant à l'essai précédent. Le premier essai de chaque tâche d'empan complexe est identique et d'une difficulté modérée (c.-à-d., 4 éléments à mémoriser à l'empan de lecture et d'opération et 3 éléments à mémoriser à l'empan de symétrie). Ensuite, la difficulté de l'essai suivant est dépendante de la proportion d'items correctement rappelés sur l'essai précédent. La taille de l'essai suivant augmente d'un élément si et seulement si le participant rappelle $100 \%$ des items mémorisés, et diminue d'un élément quand le participant rappelle moins de 
$60 \%$ des items à mémoriser. Dans le cas contraire, la taille de l'essai suivant reste la même que l'essai précédent. Chaque empan complexe comprend 6 essais. Au total, l'épreuve comprend donc 18 essais. La durée de passation est généralement de 29 minutes ( \pm 7 minutes).

Les participants réalisent chaque tâche d'empan complexe dans l'ordre suivant : 1) l'empan de lecture qui demande aux participants d'alterner entre le jugement de la véracité d'une phrase (par ex. « Les éléphants sont verts ») et la mémorisation d'un chiffre ; 2) l'empan de symétrie qui nécessite de juger la symétrie d'une figure géométrique et de mémoriser la localisation spatiale d'un carré rouge dans une grille de $4 \times 4$ cases ; 3) l'empan d'opération qui implique à la fois un jugement d'exactitude d'une opération mathématique simple (c.-à-.d. une addition ou une soustraction) et la mémorisation d'une lettre. Toutes les lettres sont des consonnes afin qu'il soit impossible de créer des syllabes. De plus, les lettres sont phonétiquement distinctes. Pour chaque tâche, le même stimulus n'apparaît jamais deux fois dans la même séquence à mémoriser.

La cotation est basée sur le nombre total de stimuli correctement rappelés de chaque tâche (Conway et al., 2005). Ce nombre total de stimuli correctement rappelés est ensuite standardisé (transformé en score z), puis la moyenne a été réalisée sur les trois tâches, de façon à réaliser un score composite de la capacité de la MDT. La proportion de réponses correctes lors de la phase interférente est également enregistrée afin de s'assurer que le traitement a été correctement réalisé durant la phase interférente. Le score de rappel d'une épreuve est exclu si et seulement si l'individu a une proportion de réponses correctes inférieure au 5e centile de la distribution; dans ce cas, le score composite est calculé à partir des deux autres épreuves (Gonthier et al., 2015). Si 2 scores de rappel sont exclus de cette façon, alors la performance du participant à l'ACCES est considérée comme non évaluable. Par ailleurs, le temps de réponse médian du traitement aux phases interférentes de chaque épreuve est également enregistré. 


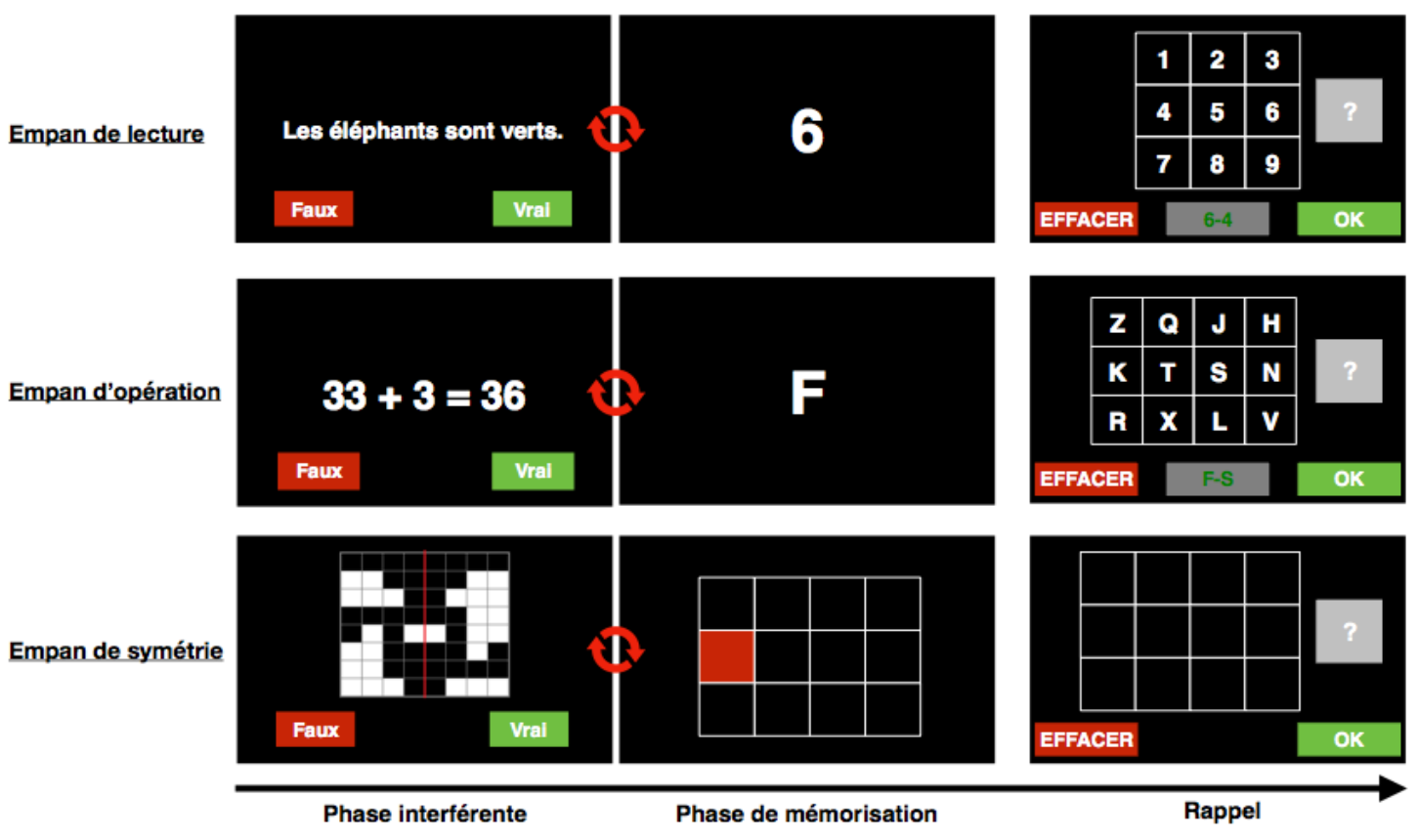

Figure 1. Illustration des 3 épreuves d'empan complexe

\section{Procédure}

Tous les participants, et leurs représentants légaux, ont été informés par écrit des objectifs de cette recherche et ont signé un consentement pour la participation volontaire à notre étude. Lors des sessions, nous demandions oralement une nouvelle fois si chaque participant était d'accord pour réaliser notre recherche. Les passations ont été effectuées collectivement soit dans une salle informatique dans les collèges et les écoles élémentaires avec l'autorisation des chefs d'établissement, soit dans une salle calme dédiée à la réalisation de l'épreuve avec des ordinateurs portables.

\section{Analyses statistiques}

Tous les traitements statistiques ont été réalisés à l'aide de R 3.5.0 (R Core Team, 2018). Les données, ainsi que le script $\mathrm{R}$ des analyses statistiques, sont disponibles sur la plateforme Open Science Framework (OSF) : http://osf.io/59aky. 
Pour l'analyse descriptive, nous avons estimé l'indice de symétrie (skewness) et l'indice d'aplatissement (kurtosis), afin d'évaluer l'importance de la violation de la normalité des données (voir Tableau 2).

Afin d'étudier le rôle de l'accroissement du temps de traitement avec l'âge sur la capacité de rappel, une analyse de similarités a été réalisée à partir de la librairie yhat (Nimon, Oswald, \& Roberts, 2013) pour déterminer la part spécifique et commune de la variance du score de rappel par deux prédicteurs, l'âge chronologique et la moyenne des temps de traitement centrés-réduits des phases interférentes (Nimon, Lewis, Kane, \& Haynes, 2008).

\section{RÉSULTATS}

Parmi notre échantillon de 753 participants, 8 participants ont été exclus de l'analyse statistique (soit 1,06\%), car ils n'avaient pas réalisé la totalité de l'évaluation de la MDT. Ensuite, 21 participants (soit 2,79\%) ont été exclus de l'analyse statistique, car la proportion de bonnes réponses aux tâches interférentes ( 2 sur 3 ) était inférieure au critère d'exclusion, c'est-à-dire au 5e centile, dans deux tâches sur trois. Le Tableau 1 présente les données sociodémographiques, le score composite de rappel et du temps de traitement de notre échantillon. Le score composite de rappel se distribue normalement (asymétrie < 1 ; aplatissement < 1) à chaque groupe d'âge (c.-à-d. l'enfance, l'adolescence et l'âge adulte), confirmant l'absence d'effet plancher ou plafond.

Tableau 1 : Description sociodémographique de l'échantillon

\begin{tabular}{|c|c|c|c|c|c|c|c|c|c|}
\hline & \multirow[b]{2}{*}{$\mathbf{n}$} & \multirow[b]{2}{*}{$\begin{array}{c}\text { Age } \\
\text { (Années) }\end{array}$} & \multirow[b]{2}{*}{$\begin{array}{l}\text { Fille } \\
(\%)\end{array}$} & \multicolumn{3}{|c|}{ Rappel } & \multicolumn{3}{|c|}{ Temps } \\
\hline & & & & $\begin{array}{c}\text { M } \\
(\mathbf{E} . T)\end{array}$ & $\begin{array}{l}\text { Asy } \\
\text { métr } \\
\text { ie }\end{array}$ & $\begin{array}{c}\text { Aplatiss } \\
\text { ement }\end{array}$ & $\begin{array}{c}\mathbf{M} \\
(\mathbf{E} . \mathbf{T})\end{array}$ & $\begin{array}{c}\text { Asy } \\
\text { métr } \\
\text { ie }\end{array}$ & $\begin{array}{c}\text { Aplatiss } \\
\text { ement }\end{array}$ \\
\hline $\begin{array}{l}\text { Enfants } \\
(8-11 \text { ans })\end{array}$ & 316 & $\begin{array}{l}10,29 \\
(1,14)\end{array}$ & 49,05 & $\begin{array}{l}-0,49 \\
(0,73)\end{array}$ & $-0,28$ & 0,48 & $\begin{array}{c}0,60 \\
(0,81)\end{array}$ & 0,60 & 0,20 \\
\hline $\begin{array}{l}\text { Adolescents } \\
(12-16 \text { ans })\end{array}$ & 231 & $\begin{array}{l}13,33 \\
(1,11)\end{array}$ & 51,95 & $\begin{array}{c}0,00 \\
(0,75)\end{array}$ & 0,01 & 0,00 & $\begin{array}{l}-0,13 \\
(0,67)\end{array}$ & 1,03 & 1,50 \\
\hline $\begin{array}{l}\text { Adultes } \\
\text { (18 - } 23 \text { ans) }\end{array}$ & 177 & $\begin{array}{l}21,16 \\
(1,02)\end{array}$ & 81,36 & $\begin{array}{c}0,80 \\
(0,49)\end{array}$ & $-0,03$ & $-0,12$ & $\begin{array}{l}-0,82 \\
(0,41)\end{array}$ & 0,61 & 0,84 \\
\hline
\end{tabular}

Notes. $\mathrm{N}=724$; Rappel : Moyenne des scores de rappel centrés-réduits des 3 épreuves ; Temps : Moyenne des temps médian des phases interférentes centrés-réduits des 3 épreuves. 
Afin d'étudier la trajectoire développementale de la capacité de la MDT des âges de 8 à 23 ans, nous avons réalisé plusieurs régressions polynomiales avec les termes linéaire, quadratique et cubique de l'âge chronologique. Tous les prérequis d'application des différentes régressions polynomiales ont été vérifiés graphiquement. La distance de Cook n'a révélé aucune valeur extrême pouvant influencer les différentes analyses.

Plusieurs modèles de régressions polynomiales ont été réalisés afin de déterminer le type de trajectoires de développement de la capacité de la MDT durant les âges de 8 à 23 ans. Quatre modèles ont été comparés : 1) le modèle constant correspond au modèle de régression où le score de rappel est prédit par une constante. Il est considéré comme le modèle de référence qui sera comparé avec les autres modèles de régression ;2) le modèle linéaire formalise le développement du score de rappel correspondant à une augmentation constante de la capacité de la MDT durant les âges de 8 à 23 ans ; 3) le modèle quadratique décrit le développement de la capacité de la MDT comme l'augmentation du score de rappel suivie d'un ralentissement lors de l'accroissement de l'âge chronologique ; 4) le modèle cubique correspond à une augmentation du score de rappel suivie d'un ralentissement pendant une période avant d'augmenter de nouveau au cours du développement.

Pour évaluer l'adéquation de chaque modèle de régression, plusieurs indicateurs ont été utilisés (Cohen, Cohen, West \& Aiken, 2013) : l'analyse de la déviance résiduelle, le critère d'information d'Akaike (AIC) et le critère d'information Bayésien (BIC). L'analyse de la déviance résiduelle permet de déterminer si la réduction des résidus entre le modèle de référence et les autres modèles de régression est significative. Les indices AIC et BIC permettent d'obtenir une information d'adéquation entre le modèle testé et les données réelles. Plus la valeur des indices AIC et BIC est petite, plus le modèle explique mieux et de manière parcimonieuse les données réelles parmi tous les modèles testés. Le Tableau 2 présente les résultats de la comparaison des modèles de régressions polynomiales. Le modèle de régression polynomiale quadratique est le meilleur modèle qui décrit la courbe développementale de la capacité de la MDT de notre échantillon, $F(2,721)=241,30$, $\mathrm{MSE}=0,63, p<0,01, R^{2}=0,401$. 
Tableau 2 : Comparaison de modèles de régression polynomiale

\begin{tabular}{lcccccc}
\hline & $\begin{array}{c}\text { ddl } \\
\text { résiduel }\end{array}$ & $\begin{array}{c}\text { Déviance } \\
\text { résiduelle }\end{array}$ & $\begin{array}{c}\text { ddl } \\
\text { différence }\end{array}$ & $\boldsymbol{F}$ & AIC & BIC \\
\hline Constant & 723 & 529,04 & & & 1831.48 & 1840.65 \\
Linéaire & 722 & 325,40 & 1 & $462,75^{* *}$ & 1481.61 & 1495.37 \\
Quadratique & 721 & 316,89 & 1 & $19,34^{* *}$ & 1464.43 & 1482.76 \\
Cubique & 720 & 316,84 & 1 & 0,11 & 1466.31 & 1489.24 \\
\hline
\end{tabular}

Note. ddl : degré de liberté ; AIC : Critère d'Information d'Akaike ; BIC : Critère d'Information Bayésien; ** $p<0,01$

Les résultats du modèle quadratique indique des effets linéaire et curviligne de l'âge chronologique sur le score de rappel $(\beta=0,75, p<0,001$ et $\beta=-0,18, p<0,001$, pour le terme linéaire et quadratique respectivement). En effet, la Figure 1 montre une importante augmentation de la capacité de la MDT avec l'âge qui ralentie vers la fin de l'adolescence et le début de l'âge adulte.

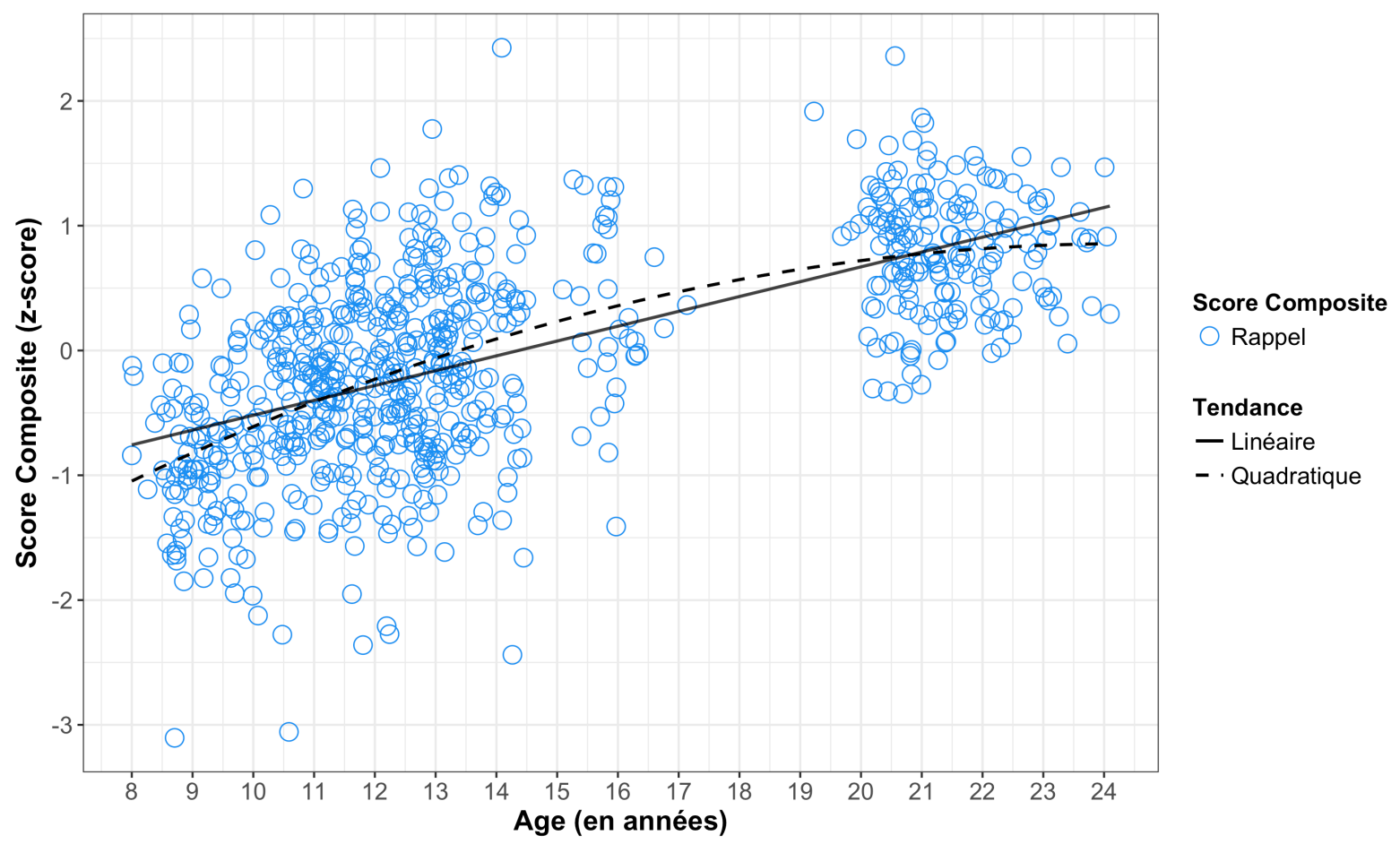

Figure 1. Distribution du score composite en fonction des groupes d'âge. 
Le temps de traitement durant la phase d'interférence est fortement lié à l'âge chronologique et au score de rappel (voir Tableau 3). En effet, le temps de traitement et l'âge chronologique expliquent 51,92 \% de l'augmentation du score de rappel, $F(2,721)=389,40$, MSE $=0,59, p<0,001$. L'analyse de similarités montre que la variance unique de l'âge chronologique explique $5,21 \%$ de l'augmentation du score de rappel, alors que celle du temps de traitement explique $13,43 \%$ de cette augmentation de performance à la tâche de MDT. La variance partagée entre l'âge chronologique et le temps de traitement explique $33,29 \%$ de la variance du score de rappel. Cela suggère que l'augmentation de la capacité de la MDT avec l'âge peut être partiellement expliquée par l'effet de l'âge sur le temps de traitement des phases interférentes.

Tableau 3 : Matrice de corrélations

\begin{tabular}{lccc}
\hline & $\mathbf{1}$ & $\mathbf{2}$ & $\mathbf{3}$ \\
\hline 1. Age & - & $0,62^{* *}$ & $-0,66^{* *}$ \\
2. Rappel & & - & $-0,68^{* *}$ \\
3. Temps & & & - \\
\hline
\end{tabular}

Note. $\mathrm{N}=724$; Rappel : Moyenne des scores de rappel centrés-réduits des 3 épreuves ; Temps : Moyenne des temps médians des phases interférentes centrés-réduits des 3 épreuves. Les valeurs de $p$ ont été corrigées par la méthode de Holm (1978). ** $p<0,01$

\section{DISCUSSION}

L'objectif de ce travail était d'illustrer, à l'aide de l'exemple de la tâche ACCES (Gonthier et al., 2017), la construction d'une mesure de la capacité de la MDT pour étudier sa trajectoire de développement. D'après la littérature, les principales contraintes à prendre en compte pour mesurer correctement la MDT sont : 1) de combiner une phase de traitement et une phase de mémorisation d'items (Conway et al., 2005) ; 2) d'informatiser son évaluation afin de mieux contrôler l'alternance de ces phases (Gonthier et al., 2015 ; Unsworth et al., 2005) ; 3) de combiner plusieurs tâches de MDT évaluant différentes modalités afin d'obtenir une estimation de l'aptitude générale de la MDT (Oswald et al., 2014) ; 4) d'utiliser une 
procédure adaptative (Gonthier et al., 2017) ; 5) d'avoir recours à une cotation partielle des résultats afin d'avoir une meilleure fiabilité de la mesure, et une meilleure qualité prédictive des performances scolaires et d'autres aptitudes cognitives comme le raisonnement fluide (Conway et al., 2005 ; Redick et al., 2012 ; St Clair-Thompson \& Sykes, 2010).

Tout d'abord, nos résultats montrent que la distribution du score composite du rappel suit approximativement une loi normale chez les enfants (8 - 11 ans), les adolescents (12 - 16 ans) et les jeunes adultes (18 - 23 ans). Cela indique qu'il n'y a pas d'effet plancher chez les individus plus jeunes ni d'effet plafond chez les individus plus âgés. La procédure adaptative de la difficulté dans une tâche de MDT constitue donc une technique simple permettant d'éviter l'effet plancher ou/et plafond dans les études développementales, même chez des individus de niveaux d'aptitude très différents. Nos résultats montrent également que la capacité de la MDT se développe de l'enfance à l'adolescence (Brocki, \& Bohlin, 2004 ; Gathercole, 1998, 1999 ; Gathercole et al., 2004, Goldstein et al., 2014 ; Thaler et al., 2013). Contrairement aux précédentes études, nos résultats indiquent que cette augmentation continue jusqu'au début de l'âge adulte où la capacité de la MDT tend à ne plus augmenter. Roberts et al. (2018) ont également trouvé des résultats similaires. Cependant, l'étendue de l'âge de notre échantillon ne dépasse pas l'âge de 23 ans. Une augmentation de la capacité de la MDT a déjà été observée au-delà de cet âge (Alloway \& Alloway, 2013 ; Swanson, 1999), ce que notre échantillon ne permet pas de mettre en évidence.

L'analyse de corrélations a montré que la capacité de rappel, l'âge chronologique et le temps de traitement, au sein des différentes phases interférentes, sont fortement corrélés. L'analyse de similarités a montré que la variance de la capacité de la MDT expliquée par l'âge est essentiellement commune avec la variance expliquée par le temps de traitement. Autrement dit, l'évolution conjointe de l'âge chronologique et du temps de traitement des phases interférentes prédit la capacité de la MDT. Concrètement, cela signifie que l'âge chronologique affecte le temps du traitement des phases interférentes qui va lui-même influencer la capacité de la MDT. Cela confirme le rôle important du temps de traitement au sein des phases interférentes dans l'évaluation de la capacité de la MDT (Barrouillet \& Camos, 2012 ; Bayliss et al., 2005 ; Saito \& Miyake, 2004 ; Towse \& Hitch, 1995). En effet, plus le temps de traitement est faible, plus le temps de rafraîchissement de la trace mnésique est élevé (Barrouillet, Bernardin, \& Camos, 2004 ; Camos \& Barrouillet, 2014). Grâce à des 
tâches comme celle présentée ici, il est possible de contrôler ce paramètre des tâches interférentes afin qu'il ne biaise pas l'analyse du psychologue clinicien concernant les performances de la MDT de l'individu (Redick et al., 2012 ; Unsworth et al., 2005), en particulier dans une approche développementale. En effet, le rôle joué par le temps passé sur la tâche interférence ne peut pas être contrôlé adéquatement sur une tâche non-informatisée qui défile au rythme du psychologue clinicien.

Il n'existe malheureusement pas d'épreuves d'empan complexe composite disponible en langue française pour les psychologues cliniciens. Il y a également une confusion entre la MCT et la MDT dans les batteries d'évaluation des aptitudes cognitives disponibles comme le WISC-V (Wechsler, 2016). Or, l'évaluation de la capacité de la MDT est une aptitude cognitive cruciale dans les apprentissages (Alloway \& Alloway, 2010 ; Barrouillet, Camos, Morlais, \& Suchaut, 2008 ; Lépine, Barrouillet, \& Camos, 2005). De plus, les tâches d'empan complexe ont l'avantage d'être de bons prédicteurs des aptitudes cognitives comme le raisonnement fluide (Ackerman, Beier, \& Boyle, 2005 ; Conway et al., 2005 ; Unsworth et al., 2009 ; Unsworth \& Engle, 2007), la compréhension langagière (Daneman \& Carpenter, 1980) ou encore la lecture et les mathématiques (Hitch, Towse, \& Hutton, 2001).

Concrètement, dans le cadre de l'accompagnement des élèves en situation de handicap, l'évaluation de la capacité de la MDT pourrait être un atout fondamental dans l'identification des troubles des apprentissages, ainsi que dans l'évaluation de l'impact des aménagements proposés. Notre étude appuie l'idée de développer et de réaliser une normalisation d'une batterie informatisée d'empan complexe multimodale adaptée pour les psychologues cliniciens. De plus, nos résultats montrent que la procédure adaptative pourrait avoir une implication clinique importante dans l'estimation de la capacité de la MDT, et donc la précision du diagnostic. En effet, elle permet d'avoir le même nombre d'essais, quels que soient les performances ou l'âge de l'individu, et d'utiliser une cotation partielle connue comme plus fiable (Conway et al., 2005 ; Redick et al., 2012) et plus adaptée dans une approche développementale (St Clair-Thompson \& Sykes, 2010), notamment pour la mise en place de programmes d'accompagnement personnalisés.

Par ailleurs, nous avons proposé un questionnaire à notre échantillon d'enfants et d'adolescents à la fin de la passation, afin de connaître leurs ressentis quant à la difficulté de la tâche. Nos jeunes participants (7 à 16 ans) ont généralement perçu l'ACCES comme une 
tâche plutôt facile, voire moyennement difficile. De plus, il n'y avait pas de différence significative au niveau de la perception de la difficulté entre les individus ayant de faibles performances en MDT (<-1,00 écart-type) par rapport à ceux ayant des performances élevées en MDT ( $>1,00$ écart-type). Ces premiers résultats ouvrent la piste à de nombreux travaux, fondamentaux comme appliqués, sur les liens entre la métacognition et la MDT. Ainsi, ce type d'épreuve est une piste intéressante permettant aux psychologues cliniciens d'obtenir une évaluation multimodale et adaptative de la capacité de la MDT.

\section{Remerciements}

Cette étude a été financée par le Conseil Régional de Picardie (CRP) et le Fonds Européen au Développement Régional (FEDER). Les auteurs tiennent à remercier tous les étudiants, les enfants, les adolescents ainsi que leur famille qui ont permis cette recherche. Les auteurs remercient Gildas Griffon, principal du collège des Fontaines, Fabien Maniak, principaladjoint du collège de Ponthieu, Cyril Douay, principal-adjoint du collège Auguste Janvier, André Leblanc, Principal du lycée agricole Le Paraclet, Isabelle Fresneau, chef d'établissement du premier degré de l'école primaire La Salle, Jean-Pierre Carron, chef d'établissement du second degré du collège La Salle, Stéphane Follet, principal et Aurélien Odiot principal-adjoint du collège Joliot Curie ainsi que Sophie Beaujouan, psychologue de l'Éducation Nationale. Les auteurs remercient également Julia Janusz, Jordan Lermont et Léo Duplenne pour leur aide dans le recueil des données. Les auteurs tiennent également à remercier chaleureusement Claire Touchet pour ses multiples relectures tout au long de la rédaction de ce manuscrit. 


\section{RÉFÉRENCES}

Ackerman, P. L., Beier, M. E., \& Boyle, M. O. (2005). Working Memory and Intelligence: The Same or Different Constructs? Psychological Bulletin, 131(1), 30-60. doi: 10.1037/0033-2909.131.1.30

Alloway, T. P. (2009). Working Memory, but Not IQ, Predicts Subsequent Learning in Children with Learning Difficulties. European Journal of Psychological Assessment, 25(2), 92-98. doi:10.1027/1015-5759.25.2.92

Alloway, T. P., \& Alloway, R. G. (2013). Working memory across the lifespan: A crosssectional approach. Journal of Cognitive Psychology, 25(1), 84-93. doi: $10.1080 / 20445911.2012 .748027$

Alloway, T. P., \& Alloway, R. G. (2010). Investigating the predictive roles of working memory and IQ in academic attainment. Journal of Experimental Child Psychology, 106(1), 20-29. doi:10.1016/j.jecp.2009.11.003

Alloway, T. P., Rajendran, G., \& Archibald, L. M. D. (2009). Working Memory in Children With Developmental Disorders. Journal of Learning Disabilities, 42(4), 372-382. doi: $10.1177 / 0022219409335214$

Alloway, T. P., Wootan, S., \& Deane, P. (2014). Investigating working memory and sustained attention in dyslexic adults. International Journal of Educational Research, 67, 11-17. doi:10.1016/j.ijer.2014.04.001

Aubry, A. \& Bourdin, B. (2017, juillet). Performance in working memory and attentional networks in gifted children. Communication présentée au 22nd Biennial World Conference : Global Perspectives in Gifted Education, Sydney, Australie. Abstract 
retrieved from https://world-gifted.org/openconf/modules/request.php? $\underline{\text { module }}=$ oc program\&action $=$ summary $\cdot p h p \& i d=148$

Baddeley, A. (2012). Working memory: theories, models, and controversies. Annual Review of Psychology, 63(1), 1-29. doi:10.1146/annurev-psych-120710-100422

Barrouillet, P., Bernardin, S., \& Camos, V. (2004). Time Constraints and Resource Sharing in Adults' Working Memory Spans. Journal of Experimental Psychology: General, 133(1), 83-100. doi:10.1037/0096-3445.133.1.83

Barrouillet, P., Bernardin, S., Portrat, S., Vergauwe, E., \& Camos, V. (2007). Time and cognitive load in working memory. Journal of Experimental Psychology: Learning, Memory, and Cognition, 33(3), 570-585. doi:10.1037/0278-7393.33.3.570

Barrouillet, P., \& Camos, V. (2012). As Time Goes By: Temporal Constraints in Working Memory. Current Directions in Psychological Science, 21(6), 413-419. doi: $10.1177 / 0963721412459513$

Barrouillet, P., \& Camos, V. (2001). Developmental Increase in Working Memory Span: Resource Sharing or Temporal Decay? Journal of Memory and Language, 45(1), 1-20. doi:10.1006/jmla.2001.2767

Barrouillet, P., Gavens, N., Vergauwe, E., Gaillard, V., \& Camos, V. (2009). Working memory span development: A time-based resource-sharing model account. Developmental Psychology, 45(2), 477-490. doi:10.1037/a0014615

Bayliss, D. M., Jarrold, C., Baddeley, A. D., \& Gunn, D. M. (2007). The relationship between short-term memory and working memory: Complex span made simple? Memory, 13(3-4), 414-421. doi:10.1080/09658210344000332 
Bayliss, D. M., Jarrold, C., Baddeley, A. D., Gunn, D. M., \& Leigh, E. (2005). Mapping the Developmental Constraints on Working Memory Span Performance. Developmental Psychology, 41(4), 579-597. doi:10.1037/0012-1649.41.4.579

Brocki, K. C., \& Bohlin, G. (2004). Executive functions in children aged 6 to 13: a dimensional and developmental study. Developmental Neuropsychology, 26(2), 571593. doi:10.1207/s15326942dn2602_3

Cain, M. K., Zhang, Z., \& Yuan, K.-H. (2017). Univariate and multivariate skewness and kurtosis for measuring nonnormality: Prevalence, influence and estimation. Behavior Research Methods, 49(5), 1716-1735. doi:10.3758/s13428-016-0814-1

Calero, M. D., García-Martín, M. B., Jiménez, M. I., Kazén, M., \& Araque, A. (2007). Selfregulation advantage for high-IQ children: Findings from a research study. Journal of Learning Disabilities, 17(4), 328-343. doi:10.1016/j.lindif.2007.03.012

Camos, V., \& Barrouillet, P. (2014). Le développement de la mémoire de travail : perspectives dans le cadre du modèle de partage temporel des ressources. Psychologie Française, 59(1), 21-39. doi:10.1016/j.psfr.2012.12.003

Camos, V., \& Barrouillet, P. (2011). Developmental change in working memory strategies: From passive maintenance to active refreshing. Developmental Psychology, 47(3), 898904. doi:10.1037/a0023193

Case, R., Kurland, D. M., \& Goldberg, J. (1982). Operational efficiency and the growth of short-term memory span. Journal of Experimental Child Psychology, 33(3), 386-404. doi:10.1016/0022-0965(82)90054-6 
Chiaravalloti, N. D., \& DeLuca, J. (2008). Cognitive impairment in multiple sclerosis. The Lancet Neurology, 7(12), 1139-1151. doi:10.1016/S1474-4422(08)70259-X

Chuderski, A. (2013). When are fluid intelligence and working memory isomorphic and when are they not? Intelligence, 41(4), 244-262. doi:10.1016/j.intell.2013.04.003

Cohen, J., Cohen, P., West, S. G., \& Aiken, L. S. (2003). Applied multiple regression/ correlation analysis for the behavioral sciences (3rd ed.). Mahwah, New Jersey: Lawrence Erlbaum.

Conklin, H. M., Luciana, M., Hooper, C. J., \& Yarger, R. S. (2007). Working memory performance in typically developing children and adolescents: behavioral evidence of protracted frontal lobe development. Developmental Neuropsychology, 31(1), 103-128. doi:10.1080/87565640709336889

Conway, A. R., Kane, M. J., Bunting, M. F., Hambrick, D. Z., Wilhelm, O., \& Engle, R. W. (2005). Working memory span tasks: A methodological review and user's guide. Psychonomic Bulletin \& Review, 12(5), 769-786. doi:10.3758/bf03196772

Cornoldi, C., \& Giofrè, D. (2014). The Crucial Role of Working Memory in Intellectual Functioning. European Psychologist, 19(4), 260-268. doi:10.1027/1016-9040/a000183

Cowan, N. (2001). The magical number 4 in short-term memory: a reconsideration of mental storage capacity. Behavioral and Brain Sciences, 24(1), 87-114. doi:10.1017/ S0140525X01003922

Delaloye, C., Ludwig, C., Borella, E., Chicherio, C., \& de Ribaupierre, A. (2008). L'Empan de lecture comme épreuve mesurant la capacité de mémoire de travail: normes basées 
sur une population francophone de 775 adultes jeunes et âgés. Revue Européenne de Psychologie Appliquée, 58(2), 89-103. doi:10.1016/j.erap.2006.12.004

Desmette, D., Hupet, M., Schelstraete, M.-A., \& Van der Linden, M. (1995). Adaptation en langue française du « Reading Span Test » de Daneman et Carpenter (1980). L'Année Psychologique, 95(3), 459-482. doi:10.3406/psy.1995.28842

Engle, R. W. (2002). Working memory capacity as executive attention. Current Directions in Psychological Science, 11(1), 19-23. doi:10.1111/1467-8721.00160

Engle, R. W., Cantor, J., \& Carullo, J. J. (1992). Individual differences in working memory and comprehension: A test of four hypotheses. Journal of Experimental Psychology: Learning, Memory, and Cognition, 18(5), 972-992. doi:10.1037/0278-7393.18.5.972

Engle, R. W., Tuholski, S. W., Laughlin, J. E., \& Conway, A. R. A. (1999). Working memory, short-term memory, and general fluid intelligence: A latent-variable approach. Journal of Experimental Psychology: General, 128(3), 309-331. doi: 10.1037//0096-3445.128.3.309

Evers, A., Muñiz, J., Bartram, D., Boben, D., Egeland, J., Fernández-Hermida, J. R., et al. (2012). Testing Practices in the 21st Century. European Psychologist, 17(4), 300-319. doi:10.1027/1016-9040/a000102

Foster, J. L., Shipstead, Z., Harrison, T. L., Hicks, K. L., Redick, T. S., \& Engle, R. W. (2014). Shortened complex span tasks can reliably measure working memory capacity. Memory \& Cognition, 43(2), 226-236. doi:10.3758/s13421-014-0461-7 
Fournet, N., Roulin, J.-L., Vallet, F., Beaudoin, M., Agrigoroaei, S., Paignon, A., et al. (2012). Evaluating short-term and working memory in older adults: French normative data. Aging \& Mental Health, 16(7), 922-930. doi:10.1080/13607863.2012.674487

Friedman, N. P., \& Miyake, A. (2004). The reading span test and its predictive power for reading comprehension ability. Journal of Memory and Language, 51(1), 136-158. doi: 10.1016/j.jml.2004.03.008

Fry, A. F., \& Hale, S. (1996). Processing Speed, Working Memory, and Fluid Intelligence: Evidence for a Developmental Cascade. Psychological Science, 7(4), 237-241. doi: 10.1111/j.1467-9280.1996.tb00366.x

Fugate, C. M., Zentall, S. S., \& Gentry, M. (2013). Creativity and Working Memory in Gifted Students With and Without Characteristics of Attention Deficit Hyperactive Disorder: Lifting the Mask. Gifted Child Quarterly, 57(4), 234-246. doi: $10.1177 / 0016986213500069$

Gathercole, S. E. (1999). Cognitive approaches to the development of short-term memory. Trends in Cognitive Sciences, 3(11), 410-419. doi:10.1016/S1364-6613(99)01388-1

Gathercole, S. E. (1998). The development of memory. Journal of Child Psychology and Psychiatry, 39(1), 3-27. doi:10.1111/1469-7610.00301

Gathercole, S. E., Pickering, S. J., Ambridge, B., \& Wearing, H. (2004). The structure of working memory from 4 to 15 years of age. Developmental Psychology, 40(2), 177190. doi:10.1037/0012-1649.40.2.177 
Gathercole, S. E., Pickering, S. J., Knight, C., \& Stegmann, Z. (2003). Working memory skills and educational attainment: evidence from national curriculum assessments at 7 and 14 years of age. Applied Cognitive Psychology, 18(1), 1-16. doi:10.1002/acp.934

Goldstein, G., Allen, D. N., Thaler, N. S., Luther, J. F., Panchalingam, K., \& Pettegrew, J. W. (2014). Developmental aspects and neurobiological correlates of working and associative memory. Neuropsychology, 28(4), 496-505. doi:10.1037/neu0000053

Gonthier, C., Aubry, A., \& Bourdin, B. (2017). Measuring working memory capacity in children using adaptive tasks: Example validation of an adaptive complex span. Behavior Research Methods, 27(8), 1-12. doi:10.3758/s13428-017-0916-4

Gonthier, C., Thomassin, N., \& Roulin, J.-L. (2015). The composite complex span: French validation of a short working memory task. Behavior Research Methods, 48(1), 233242. doi:10.3758/s13428-015-0566-3

Hitch, G. J., Towse, J. N., \& Hutton, U. (2001). What limits children's working memory span? Theoretical accounts and applications for scholastic development. Journal of Experimental Psychology: General, 130(2), 184-198. doi: $10.1037 / 0096-3445.130 .2 .184$

Holm, S. (1978). A Simple Sequentially Rejective Multiple Test Procedure. Scandinavian Journal of Statistics, 6(2), 65-70.

Hutton, U. M. Z., \& Towse, J. N. (2001). Short-term memory and working memory as indices of children's cognitive skills. Memory, 9(4-6), 383-394. doi: $10.1080 / 09658210042000058$ 
Kail, R. (2002). Developmental Change in Proactive Interference. Child Development, 73(6), 1703-1714. doi:10.1111/1467-8624.00500

Kane, M. J., \& Engle, R. W. (2000). Working-memory capacity, proactive interference, and divided attention: Limits on long-term memory retrieval. Journal of Experimental Psychology: Learning, Memory, and Cognition, 26(2), 336-358. doi10.1037//0278-7393.26.2.336

Kaufman, A. S., \& Kaufman, N. L. (2008). KABC-II : Batterie pour l'examen psychologique de l'enfant - deuxième édition. Paris : Édition du Centre de Psychologie Appliquée.

Korkman, M., Kirk, U. \& Kemp, S. (2012). NEPSY-II : Bilan neuropsychologique de l'enfant-seconde édition. Paris : Édition du Centre de Psychologie Appliquée.

Lee, J., \& Park, S. (2005). Working Memory Impairments in Schizophrenia: A MetaAnalysis. Journal of Abnormal Psychology, 114(4), 599-611. doi:10.1037/0021-843X. 114.4.599

Lépine, R., Barrouillet, P., \& Camos, V. (2005). What makes working memory spans so predictive of high-level cognition? Psychonomic Bulletin \& Review, 12(1), 165-170.

Linck, J. A., Osthus, P., Koeth, J. T., \& Bunting, M. F. (2014). Working memory and second language comprehension and production: a meta-analysis. Psychonomic Bulletin \& Review, 21(4), 861-883. doi:10.3758/s13423-013-0565-2

Logie, R. H. (2011). The Functional Organization and Capacity Limits of Working Memory. Current Directions in Psychological Science, 20(4), 240-245. doi: $10.1177 / 0963721411415340$ 
Loosli, S. V., Rahm, B., Unterrainer, J. M., Weiller, C., \& Kaller, C. P. (2014). Developmental Change in Proactive Interference Across the Life Span: Evidence From Two Working Memory Tasks. Developmental Psychology, 50(4), 1060-1072. doi:10.1037/a0035231

Lustig, C., May, C. P., \& Hasher, L. (2001). Working memory span and the role of proactive interference. Journal of Experimental Psychology: General, 130(2), 199-207. doi: $10.1037 / / 0096-3445.130 .2 .199$

Luo, D., Thompson, L. A., \& Detterman, D. K. (2006). The Criterion Validity of Tasks of Basic Cognitive Processes. Intelligence, 34(1), 79-120. doi:10.1016/j.intell. 2004.12.003

Maehler, C., \& Schuchardt, K. (2009). Working memory functioning in children with learning disabilities: does intelligence make a difference? Journal of Intellectual Disability Research, 53(1), 3-10. doi:10.1111/j.1365-2788.2008.01105.x

Martinussen, R., Hayden, J., Hogg-Johnson, S., \& Tannock, R. (2005). A Meta-Analysis of Working Memory Impairments in Children With Attention-Deficit/Hyperactivity Disorder. Journal of the American Academy of Child \& Adolescent Psychiatry, 44(4), 377-384. doi:10.1097/01.chi.0000153228.72591.73

McCabe, D. P. (2010). The influence of complex working memory span task administration methods on prediction of higher level cognition and metacognitive control of response times. Memory \& Cognition, 38(7), 868-882. doi:10.3758/MC.38.7.868

McCoach, D. B., Rambo, K. E., \& Welsh, M. (2012). Assessing the Growth of Gifted Students. Gifted Child Quarterly, 57(1), 56-67. doi:10.1177/0016986212463873 
Nimon, K., Oswald, F., \& Roberts, K. J. (2013). yhat: Interpreting Regression Effects (version 2.0-0). [Computer software]. Retrieved from https:/CRAN.R-project.org/ package $=$ yhat

Numminen, H., Service, E., Ahonen, T., \& Ruoppila, I. (2001). Working memory and everyday cognition in adults with Down's syndrome. Journal of Intellectual Disability Research, 45(2), 157-168. doi:10.1046/j.1365-2788.2001.00298.x

Oswald, F. L., McAbee, S. T., Redick, T. S., \& Hambrick, D. Z. (2014). The development of a short domain-general measure of working memory capacity. Behavior Research Methods, 47(4), 1343-1355. doi:10.3758/s13428-014-0543-2

Peirce, J. W. (2007). PsychoPy_Psychophysics software in Python. Journal of Neuroscience Methods, 162(1), 8-13. doi:10.1016/j.jneumeth.2006.11.017

Redick, T. S., Broadway, J. M., Meier, M. E., Kuriakose, P. S., Unsworth, N., Kane, M. J., \& Engle, R. W. (2012). Measuring Working Memory Capacity With Automated Complex Span Tasks. European Journal of Psychological Assessment, 28(3), 164-171. doi: $10.1027 / 1015-5759 / \mathrm{a} 000123$

Redick, T. S., Shipstead, Z., Meier, M. E., Montroy, J. J., Hicks, K. L., Unsworth, N., et al. (2016). Cognitive predictors of a common multitasking ability: Contributions from working memory, attention control, and fluid intelligence. Journal of Experimental Psychology: General, 145(11), 1473-1492. doi:10.1037/xge0000219

Roberts, K. L., Strait, J. A. E., \& Decker, S. L. (2018). Developmental Trajectories of Verbal, Static Visual-Spatial, and Dynamic Visual-Spatial Working Memory. Contemporary School Psychology, 106(1), 1-10. doi:10.1007/s40688-018-0176-z 
Rosseel, Y. (2012). lavaan: An R Package for Structural Equation Modeling. Journal of Statistical Software, 48(2). doi:10.18637/jss.v048.i02

Saito, S., \& Miyake, A. (2004). On the nature of forgetting and the processing-storage relationship in reading span performance. Journal of Memory and Language, 50(4), 425-443. doi:10.1016/j.jml.2003.12.003

Siegel, L. S., \& Ryan, E. B. (1989). The Development of Working Memory in Normally Achieving and Subtypes of Learning Disabled Children. Child Development, 60(4), 973-978. doi:10.2307/1131037

Seigneuric, A., De Guibert, M., Megherbi, H., Potier, D., \& Picard, A. (2009). La MTVE : une épreuve de mémoire de travail verbale chez l'enfant adaptée du Listening Span Task de Siegel et Ryan (1989). L'Année Psychologique, 108(02), 273-308. doi:10.4074/ S0003503308002042

St Clair-Thompson, H. L. (2007). The influence of strategies on relationships between working memory and cognitive skills. Memory, 15(4), 353-365. doi: $10.1080 / 09658210701261845$

St Clair-Thompson, H. L., \& Allen, R. J. (2013). Are forward and backward recall the same? A dual-task study of digit recall. Memory \& Cognition, 41(4), 519-532. doi:10.3758/ s13421-012-0277-2

St Clair-Thompson, H., \& Sykes, S. (2010). Scoring methods and the predictive ability of working memory tasks. Behavior Research Methods, 42(4), 969-975. doi:10.3758/ BRM.42.4.969 
Swanson, H. L. (1999). What develops in working memory? A life span perspective. Developmental Psychology, 35(4), 986-1000. doi:10.1037/0012-1649.35.4.986

Tamnes, C. K., Walhovd, K. B., Grydeland, H., Holland, D., Østby, Y., Dale, A. M., \& Fjell, A. M. (2013). Longitudinal Working Memory Development Is Related to Structural Maturation of Frontal and Parietal Cortices. Journal of Cognitive Neuroscience, 25(10), 1611-1623. doi:10.1162/jocn_a_00434

Thaler, N. S., Goldstein, G., Pettegrew, J. W., Luther, J. F., Reynolds, C. R., \& Allen, D. N. (2013). Developmental aspects of working and associative memory. Archives of Clinical Neuropsychology, 28(4), 348-355. doi:10.1093/arclin/acs114

Towse, J. N., \& Hitch, G. J. (1995). Is there a Relationship between Task Demand and Storage Space in Tests of Working Memory Capacity? The Quarterly Journal of Experimental Psychology Section A, 48(1), 108-124. doi:10.1080/14640749508401379

Towse, J. N., Hitch, G. J., \& Hutton, U. (1998). A Reevaluation of Working Memory Capacity in Children. Journal of Memory and Language, 39(2), 195-217. doi:10.1006/ jmla.1998.2574

Unsworth, N., \& Engle, R. W. (2007). On the division of short-term and working memory: An examination of simple and complex span and their relation to higher order abilities. Psychological Bulletin, 133(6), 1038-1066. doi:10.1037/0033-2909.133.6.1038

Unsworth, N., Heitz, R. P., Schrock, J. C., \& Engle, R. W. (2005). An Automated Version of the Operation Span Task. Behavior Research Methods, 37(3), 498-505. doi:10.3758/ BF03192720 
Van Viersen, S., Kroesbergen, E. H., Slot, E. M., \& de Bree, E. H. (2016). High Reading Skills Mask Dyslexia in Gifted Children. Journal of Learning Disabilities, 49(2), 189199. doi:10.1177/0022219414538517

Vock, M., \& Holling, H. (2008). The measurement of visuo-spatial and verbal-numerical working memory: Development of IRT-based scales. Intelligence, 36(2), 161-182. doi: 10.1016/j.intell.2007.02.004

Wechsler, D. (2016). Manuel de l'échelle d'intelligence de Wechsler pour enfants (5e éd.). Paris : Éditions du Centre de Psychologie Appliquée.

Weiss, D. J. (1982). Improving Measurement Quality and Efficiency with Adaptive Testing. Applied Psychological Measurement, 6(4), 473-492. doi: $10.1177 / 014662168200600408$

Wilhelm, O., Oberauer, K., Hildebrandt, A., \& Conway, A. (2013). What is working memory capacity, and how can we measure it? Frontiers in Psychology, 4, 1-22. doi:10.3389/ fpsyg.2013.00433

Wu, K. K., Chan, S. K., Leung, P. W. L., Liu, W.-S., Leung, F. L. T., \& Ng, R. (2011). Components and developmental differences of executive functioning for school-aged children. Developmental Neuropsychology, 36(3), 319-337. doi: $10.1080 / 87565641.2010 .549979$ 